\title{
Spectropolarimetry of Type IIn SN2010j1: Peering Into the Heart of a Monster
}

\author{
Franz E. Bauer ${ }^{1,2,3}$, Paula Zelaya ${ }^{1,3}$, Alejandro Clocchiatti ${ }^{1,3}$, and \\ Justyn Maund ${ }^{4}$ \\ ${ }^{1}$ Pontificia Universidad Católica de Chile, Departamento de Astronomía y Astrofísica, Casilla \\ 306, Santiago 22, Chile ${ }^{2}$ Space Science Institute, 4750 Walnut Street, Suite 205, Boulder, \\ Colorado $80301{ }^{3}$ Millennium Center for Supernova Science (MCSS), ${ }^{4}$ Astrophysics Research \\ Centre, School of Mathematics and Physics, Queen's University Belfast, Belfast BT7 1NN, UK

\begin{abstract}
We report results for two epochs of spectropolarimetry on the luminous type IIn SN2010j1, taken at $\approx 36$ and 85 days post-explosion with VLT FORS2-PMOS. The high signalto-noise data demonstrate distinct evolution in the continuum and the broad lines point to a complex origin for the various emission components and to a potentially common polarization signal for the type IIn class even over 1-2 orders of magnitude in luminosity output.
\end{abstract}

Keywords. (stars:) supernovae: individual (SN2010jl), techniques: spectroscopic,polarimetric

\section{Introduction}

SN2010jl was discovered on Nov 3.52 UT, 2010 with a broad visual magnitude of 12.9 (CBET 2532) and was later isolated to have an explosion date prior to Oct 9.60 UT, 2010 (Stoll et al. 2011). It was spectroscopically classified as a prompt stronglyinteracting type IIn (CBET 2536), and is ultraviolet- and X-ray-bright, as expected for its class (ATEL 3012). The SN resides in the metal-poor UGC 5189A at 48.9 Mpc (ATEL 3010 ), giving it a peak uncorrected $M_{\mathrm{V}}=-20.55$, roughly 1-3 mags more luminous than typical IIn $\left(M_{\mathrm{V}}=-17\right.$ to -19.3 ; Richardson et al. 2002; Kiewe et al. 2012). Type IIn $\mathrm{SNe}$ are characterized by intense, composite-profile emission lines, a lack of the broad P-Cygni absorption troughs, and X-ray/radio emission. The properties are explained as arising from a strong interaction between the fast-moving SN ejecta and a dense, slowmoving CSM, created by significant and likely rapid mass loss of the progenitor star (e.g., Chugai \& Danziger 1994). Yet many questions remain regarding the mass loss history of these progenitors, the geometry of both the explosion and CSM, and the origin of the broad emission lines (e.g., Chugai 2001). The geometrical insight gained from spectropolarimetry may allow a critical approach to these open issues, especially for rare luminous type IIn's like SN2010jl, which could provide extreme tests of models. The only other type IIn SNe to be observed in polarized light were SN1998S (Leonard et al. 2000; Wang et al. 2001) and SN1997eg (Hoffman et al. 2008) to provide points of comparison.

\section{Data}

We obtained $1 \mathrm{hr}$ spectropolarimetric integrations of SN2010jl on Nov. 14.3 UT, 2010 (E1) and Jan. 2.2 UT, 2011 (E2) with FORS2 PMOS on the 8m VLT-UT1. These correspond to 35.7 and 84.6 days after earliest detection. SN2010jl was also observed via spectropolarimetry by Patat et al. 2011 (hereafter P11) at lower S/N (2.7hr) and spectral resolution on Nov. 18.2 UT, 2010 with the Calar Alto $2 \mathrm{~m}$ telescope. A 3rd epoch observed on days $\approx 530-540$ is still being analyzed. Spectral reductions, calibrations, and error estimates were performed following the prescriptions described in Patat \& Romaniello (2006). Following P11, we assume negligible $(<0.3 \%)$ interstellar polarization (ISP); this 
is consistent with the nearly complete depolarization seen in narrow $\mathrm{H} \alpha$ at both epochs, despite a $0.5 \%$ change in continuum polarization.

\section{Results and Discussion}

Our high S/N spectra provide several useful insights into the properties of SN2010jl. The E1 spectrum provides a significant improvement upon the Nov 18, 2010 spectrum of P11, and confirms many of their findings, while the E2 spectrum allows us to understand the evolution of the various polarized components. Here are our main findings:

- The narrow $\mathrm{H} \alpha$ lines presumably stem from the slow-moving and possibly clumpy circumstellar wind material outside of the SN shock and polarized regions. This recombination region provides a large electron scattering shell to naturally explain the various observed polarization signals and narrow-line depolarization.

- The continuum is strongly polarized, and is stronger toward the blue. Values of $1.7-$ $2.5 \%$ on E1 and $1.2-2.0 \%$ on E2 imply axial ratios of $\lesssim 0.7$ and $\lesssim 0.8$ for the continuumgenerating region, respectively. On average, $Q_{\text {cont }} \approx 0.0 \%$ with no apparent evolution, while $U_{\text {cont }} \approx 2.0 \%$ on E1, decreasing to $\sim 1.5 \%$ by E2, implying a clear axis to the polarized signal and presumed continuum asymmetry, which is slowly becoming more isotropic. The polarized continuum fades faster than the actual continuum, particularly in the blue, while the strength of total and polarized broad $\mathrm{H} \alpha$ does not appear to evolve, indicating that these components originate from distinct regions.

- No obvious line features are seen in polarization percentage, although broad $\mathrm{H} \alpha$ and $\mathrm{H} \beta$ lines are clearly distinguished in polarized flux. Other line complexes are presumably strongly polarized as well, since they are individually distinct in Stokes $Q, U$, and polarization angle plots. Together, this implies strong depolarization is present in most lines at both epochs, presumably due to continuum dilution. Continuum-subtracted polarization percentages (and hence asymmetries) for the lines are generally higher than the nominal continuum values reported above. The variations in the evolving polarization strength and angle for line emission of broad $\mathrm{H}, \mathrm{He}$, and Fe implies that the various elements likely arise in somewhat distinct asymmetric regions: $\mathrm{H}$ and He from shocked CSM possibly associated with swept up material from the SN shock, and Fe from fast-moving shocked ejecta.

\section{Acknowledgments}

We gratefully thank the Programa de Financiamiento Basal, Iniciativa Cientifica Milenio grant P10-064-F (MCSS), with input from "Fondo de Innovación para la Competitividad, del Ministerio de Economía, Fomento y Turismo de Chile", FONDECYT Regular \#1101024 and Beca de Doctorado, FONDAP-CATA 15010003, and the Royal Society.

\section{References}

Chugai, N. N. 2001, MNRAS, 326, 1448

Chugai, N. N. \& Danziger, I. J. 1994, MNRAS, 268, 173

Hoffman, J. L., et al. 2008, ApJ, 688, 1186

Kiewe, M., et al. 2012, ApJ, 744, 10

Leonard, D. C., Filippenko, A. V., Barth, A. J., \& Matheson, T. 2000, ApJ, 536, 239

Patat, F. \& Romaniello, M. 2006, PASP, 118, 146

Patat, F., et al. 2011, A\&SA, 527, L6 (P11)

Richardson, D., et al. 2002, AJ, 123, 745

Stoll, R., et al. 2011, ApJ, 730, 34

Wang, L., Howell, D. A., Höflich, P., \& Wheeler, J. C. 2001, ApJ, 550, 1030 